\title{
Computational Investigation of Experimental Interaction Impedance Obtained by Perturbation for Helical Traveling-Wave Tube Structures
}

\author{
Carol L. Kory \\ ANALEX Corporation/NASA Lewis Research Center \\ 21000 Brookpark Road, MS 54-5 \\ Cleveland, Ohio 44135 \\ James A. Dayton, Jr. \\ NASA Lewis Research Center \\ Cleveland, Ohio 44135
}

\begin{abstract}
Conventional methods used to measure the cold-test interaction impedance of helical slow-wave structures involve perturbing a helical circuit with a cylindrical dielectric rod placed on the central axis of the circuit. It has been shown that the difference in resonant frequency or axial phase shift between the perturbed and unperturbed circuits can be related to the interaction impedance. However, because of the complex configuration of the helical circuit, deriving this relationship involves several approximations. With the advent of accurate threedimensional helical circuit models $[1,2$, and 3$]$, these standard approximations can be fully investigated. This paper addresses the most prominent approximations made in the analysis for measured interaction impedance by Lagerstrom [4] and investigates their accuracy using the three-dimensional simulation code MAFIA. It is shown that a more accurate value of interaction impedance can be obtained by using three-dimensional computational methods rather than performing costly and time consuming experimental cold-test measurements.
\end{abstract}

\section{INTRODUCTION}

Obtaining slow-wave circuit interaction impedance is an important part of the design process for a traveling-wave tube (TWT) as this parameter is related to the gain and efficiency of 
the tube or the minimum length of a backward-wave oscillator. On-axis interaction impedance is proportional to the strength of coupling between the RF wave and the electron beam, defined for the $n^{\text {th }}$ RF space harmonic [5] as

$$
K_{n}=\frac{E_{z n}^{2}(0)}{2 \beta_{n}^{2} P}
$$

where $E_{z x}(0)$ is the on-axis longitudinal electric field magnitude of the $n^{\text {th }}$ axial space harmonic, $P$ is the time averaged RF power flow and $\beta_{n}$ is the axial phase constant of the $n^{\text {th }}$ space harmonic defined by

$$
\beta_{n}=\beta+\frac{2 \pi n}{L}
$$

where $\beta$ is the fundamental axial phase constant and $\mathrm{L}$ is the pitch length.

Synchronism with the beam for the electromagnetic wave propagating on the helix is desirable for the fundamental RF space harmonic; thus, we need to calculate the fundamental ( $\mathrm{n}$ $=0$ ) harmonic term in equation 1 to determine the on-axis irteraction impedance which is related to the gain of the circuit and is used as an input in various TWT modeling codes such as [6]. To determine the interaction impedance of the backward wave mode which is related to backward gain (backward wave oscillations) we need to calculate the impedance associated with the $n=-1$ space harmonic is required.

Typically, interaction impedance is obtained by performing cold-tests on the helical circuit or a scale model of the circuit. This experimental procedure is time-consuming and expensive, but until recently it was thought to be the most arcurate means of determining interaction impedance. Most often, measurements on helical circuits are performed by using 
nonresonant perturbation techniques where the axial phase shift is measured at a certain excitation frequency for a perturbed and an unperturbed circuit. After some lengthy algebraic manipulation, Lagerstrom shows that this difference in phase shift, $\Delta \beta$, is proportional to the perturbed and unperturbed electric fields integrated over the perturbing rod volume divided by the power within the structure (equation 3.18 of [4])

$$
\Delta \beta=\frac{\omega \varepsilon_{o}\left(\varepsilon^{\prime}-1\right) \int_{\Delta V} \mathrm{E}^{\prime} \cdot \mathrm{E}^{*} d v}{4 P L} .
$$

$\mathbf{E}^{*}$ denotes the complex conjugate of the original electric field and $\mathbf{E}^{\prime}$ denotes the perturbed field. If representations of the unperturbed and perturbed fields are known, equation 3 can be solved providing an expression relating this measurable quantity to the interaction impedance.

However, because of the complexity of the helical structure, several approximations become necessary in representing $\mathbf{E}^{*}$ and $\mathbf{E}^{\prime}$. It is well known that these assumptions will cause some error in the measurement, and several researchers have addressed this issue in an attempt to improve upon Lagerstrom's work [7], [8]. Nevertheless, several approximations are still involved making direct calculation of equation 1 with a three-dimensional computer code $[1,2$, and 3] the most accurate method.

An accurate, three-dimensional, computational helical cold-test model has been developed and reported on in [1]. This model allows, for the first time, a complete investigation of the conventional approximations which go into helical circuit analysis. A summary will be given of Lagerstrom's analysis emphasizing the major assumptions made, followed by the results from an investigation of each of these assumptions using MAFIA. MAFIA (Solution of MAxwell's equations by the Finite-Integration-Algorithm) is a powerful, electrodynamic code 
that is used for computer-aided design of two-dimensional and fully three-dimensional electromagnetic devices $[9,10]$.

In addition to the approximations that go into the experimental impedance calculations, there are several human factors which can cause inaccurate measurements. For example, the perturbing rod may be displaced from the central axis of the circuit slightly, or the dielectric constant of the perturbing rod may be uncertain. Also, reflections may be present because of mismatches at the input/output couplers, nonuniformity in the helical pitch, or because of other manufacturing errors.

The circuit used as a model for this study is the helix slow-wave circuit from a 40 Watt, 18-40 GHz TWT for the millimeter-wave power module (MMPM) [11]. The experimental circuit includes a rectangular, tungsten, helical tape supported by $\mathrm{T}$-shaped $\mathrm{BeO}$ rods inside a conducting barrel as shown for the end view in Figure 1. The specific results presented here apply only to the mentioned circuit; however, these results can serve as a general guide for similar devices, and the computational techniques are readily applicable to other TWT's. 


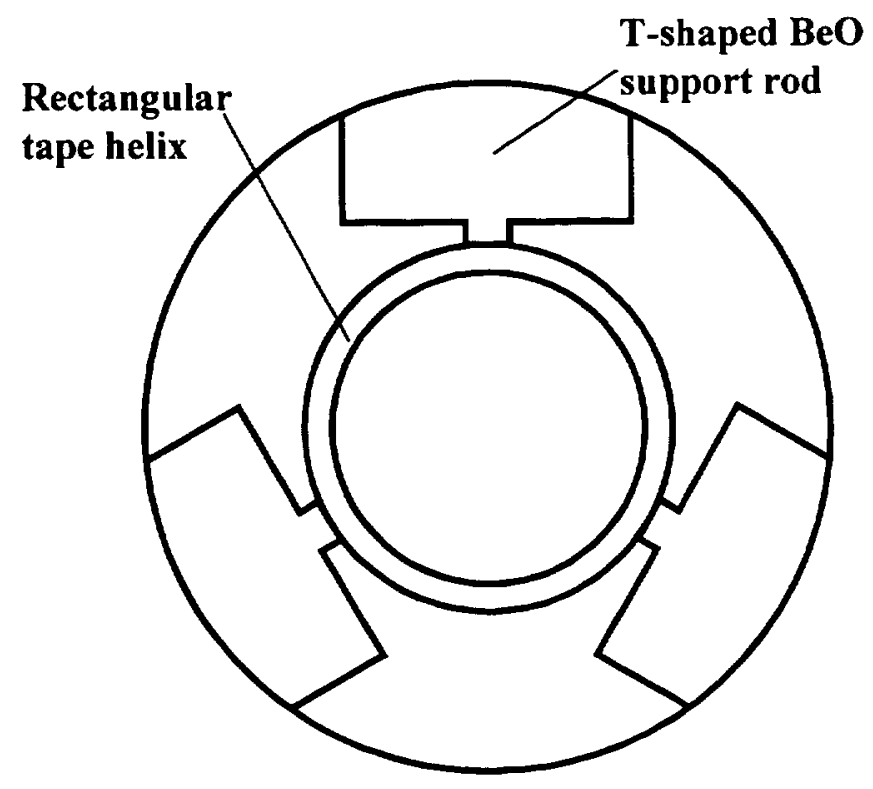

Figure 1 Hughes MMPM helical slow-wave circuit

\section{THEORY OF INTERACTION IMPEDANCE MEASUREMENTS [4]}

Lagerstrom uses the perturbation method to distort original fields in the circuit in order to gain knowledge about the strength of the fields in the region of interaction. Reviewing the perturbation formula of equation 3 , we recognize that it is necessary to derive expressions for unperturbed and perturbed electric fields within the perturbation rod volume. If these expressions can be derived in terms of the magnitude of the $\mathrm{n}^{\text {th }}$ axial space harmonic of the onaxis longitudinal electric field, $E_{m}(0)$, the interaction impedance can be expressed in terms of the measurable quantity $\Delta \beta$. Thus, as a basis for the helical field configuration we start by considering a circular aperture with radius a which identically repeats itself every distance $L$ in the axial direction. The space harmonic nature of the field solutions is such that any field quantity can be written as 


$$
e^{-j \beta z} \sum_{n=-\infty}^{\infty} a_{n} e^{-j \frac{2 \pi n}{L} z}
$$

where the time variation $\mathrm{e}^{\mathrm{j} \omega \mathrm{t}}$ is understood and $\mathrm{a}_{n}$ is the $\mathrm{n}^{\text {th }}$ Fourier amplitude coefficient which is a function of $\mathrm{r}$ and $\theta$ only. Inside a homogeneous region of a circular aperture in free space, the space harmonic components are solutions of the homogeneous wave equation in cylindrical coordinates. So, the longitudinal electric field can be expressed as

$$
\hat{E}_{z}(r, \theta, z)=e^{-j \beta z} E_{z}(r, \theta, z)=e^{-j \beta z} \sum_{v, n=-\infty}^{\infty}\left[a_{v n} I_{v}\left(\gamma_{n} r\right)+b_{v n} K_{v}\left(\gamma_{n} r\right)\right] e^{j v \theta} e^{-j \frac{2 \pi n}{L} z}
$$

where $I_{v}\left(\gamma_{n} r\right)$ and $K_{v}\left(\gamma_{n} r\right)$ are the modified Bessel functions of the first and second kind and $\gamma_{n}$ is the radial propagation constant of the $\mathrm{n}^{\text {th }}$ space harmonic defined by

$$
\gamma_{n}=\sqrt{\beta_{n}^{2}-k^{2}}, \quad k^{2}=\omega^{2} \mu_{o} \varepsilon_{o}
$$

where $\omega$ is the radian frequency $2 \pi f$, and $\mu_{0}$ and $\varepsilon_{0}$ are the free space permeability and permittivity, respectively. When the axis is included in the circular aperture, $K_{v}\left(\gamma_{n} r\right)$ cannot represent a physical field, forcing $b_{v n}$ to equal zero. Thus, in the region $0 \leq r \leq a$

$$
\hat{E}_{z}(r, \theta, z)=e^{-j \beta z} E_{z}(r, \theta, z)=e^{-j \beta z} \sum_{\nu, n=-\infty}^{\infty} E_{z v n} I_{\nu}\left(\gamma_{n} r\right) e^{j v \theta} e^{-j \frac{2 \pi n}{L} z}
$$

The phase velocity $\mathrm{v}_{\mathrm{p} n}$ of the $v n^{\text {th }}$ space harmonic is given by $\mathrm{v}_{\mathrm{p} n}=\frac{\omega}{\beta_{n}}$. The interaction impedance for the $v n^{\text {th }}$ space harmonic at a non-zero value o: radius can be defined as $K_{m}(r)=\frac{E_{z m}(r) E_{z m}^{*}(r)}{2 \beta_{n}^{2} P}$ where $\mathrm{P}$ denotes the time-averaged real power flowing in the mode 
of which this $v n^{\text {th }}$ space harmonic is a part. Since there can be no azimuthal variation on the axis of a circular aperture, the interaction impedance at $r=0$ reduces to equation 1 .

Although at any position all of the space harmonics are present and contributing to the total field, the perturbing rod can be strategically placed in a region where the space harmonic of interest is dominant. It is obvious that on the axis of a circular aperture, the $\theta$-varying space harmonics vanish $(v=0)$ and the $n=0$ space harmonic will dominate. If a dielectric rod of infinitesimal diameter were placed on the axis, only this space harmonic would be present in the region of perturbation. Accordingly, only this space harmonic would be involved in the perturbation integral of equation 3 making the problem straightforward. An infinitesimally thick rod is, of course, not physically possible, and in practice the rod must be thick enough to produce a measurable perturbation. Therefore, other space harmonics will be present over the rod volume making the problem far more complicated. In [4], the perturbing rod is assumed to be small enough in diameter that the space harmonics other than $v=n=0$ can be neglected over the entire perturbation volume. Another assumption made is that the TM portion of the fields dominates the TE portion. Therefore, electric fields can be expressed in terms of the axial and radial components only, neglecting the azimuthal component.

The approach used in [4] to calculate the local fields for the perturbation integral considers that the perturbation rod is placed in a region containing a "snap-shot" of the original field configuration. Lagerstrom refers to this as the "incident" field. The "induced" or "reflected" field, $\Delta \mathrm{E}$, is calculated as the additional field needed to satisfy the perturbing rod boundary conditions. With these calculated, $E^{\prime}$ can be calculated for use in equation 3 . Lagerstrom justifies this procedure as follows: the propagation constant is changed only slightly 
by the addition of the dielectric rod, and since the media of the slow-wave circuit determines the unperturbed propagation constant, the fields at the perturbat on would be expected to change only slightly as well.

Applying Lagerstrom's approximations that only the $\nu=n=0$ space harmonic will be present and the TE portion of fields can be neglected within the perturbation volume, and the perturbed and unperturbed fields are equal outside the perturbation rod, we proceed in solving equation 3. To express the fields in the actual structure, [4] begins as if to solve the boundary value problem of a perturbing rod of radius $b$ in a sheath helix of average radius a and axial turn length $\mathrm{L}$. Since the particular configuration of the circuit is not involved, the local field solutions at the rod and the evaluated integral should apply for an arbitrary structure with a dominant nonazimuthally varying harmonic.

The TE and TM fields can be expressed separately in terms of the longitudinal fields, Ez and $\mathrm{Hz}$. Using the notation $\hat{\mathbf{E}}=e^{-j \beta z} \mathbf{E}$ where $\mathbf{E}$ is now a function of r only, Maxwell's equations [12] are used to give

$$
\begin{aligned}
& T M: E_{\boldsymbol{r}}=\frac{j \beta}{\gamma^{2}} \frac{\partial}{\partial} E_{z}, H_{\theta}=j \frac{\omega \varepsilon_{r}}{\gamma^{2}} \frac{\partial}{\partial} E_{z} \\
& T E: H_{\boldsymbol{r}}=\frac{j \beta}{\gamma^{2}} \frac{\partial}{\partial} H_{z}, E_{\theta}=-j \frac{\omega u}{\gamma^{2}} \frac{\partial}{\partial} H_{z}
\end{aligned}
$$

From equation 7 , the unperturbed longitudinal electric field in the region inside the helix, $0 \leq \mathrm{r} \leq$ a, can be expressed as

$$
\boldsymbol{E}_{\tau}=A I_{0}(\gamma r)
$$


Since $I_{0}(\gamma r)=1$ on the axis, A is the magnitude of the axial electric field on the axis, $E_{z}(0)=A$. From equation 8 , the transverse fields needed in the perturbation integral of equation 3 associated with the TM portion of the fields can be derived:

$$
E_{r}=j \frac{\beta}{\gamma} A I_{1}(\gamma r)
$$

Next, a cylindrical dielectric rod of radius $b$ and dielectric constant $\varepsilon^{\prime} \varepsilon_{o}$ is placed on the helical axis, consequently creating a new boundary condition. Two regions now exist, I, the region between the rod and the helical conductor and II, the region within the dielectric rod (See Figure 2).

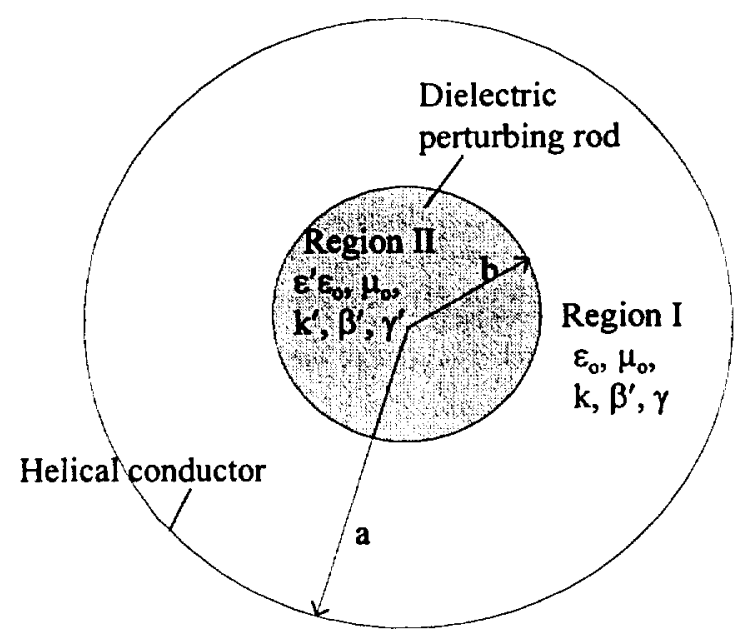

\section{Figure 2 Helical perturbation rod model}

Using equations 5 and 8 , the fields in the two regions can be expressed as

Region I $(b \leq r \leq a)$ : 


$$
\begin{aligned}
& \boldsymbol{E}_{z I}=A_{I} I_{0}(\gamma r)+B_{I} K_{0}(\gamma r) \\
& \boldsymbol{E}_{\boldsymbol{r} I}=j \frac{\beta^{\prime}}{\gamma}\left[A_{I} I_{1}(\gamma r)-B_{I} K_{1}(\gamma r)\right] \\
& \gamma^{2}=\beta^{2}-k^{2} \quad k^{2}=\omega^{2} \mu_{o} \varepsilon_{o}
\end{aligned}
$$

Region II $(0 \leq \mathrm{r} \leq \mathrm{b})$ :

$$
\begin{aligned}
& E_{z I I}=A_{I I} I_{0}\left(\gamma^{\prime} r\right) \\
& E_{r I I}=j \frac{\beta^{\prime}}{\gamma^{\prime}} A_{I I} I_{1}\left(\gamma^{\prime} r\right) \\
& \gamma^{\prime 2}=\beta^{\prime 2}-k^{\prime 2}, k^{\prime 2}=\varepsilon^{\prime} k^{2}
\end{aligned}
$$

For the perturbed case, the magnitude of the longitudinal field on the axis is

$E_{z I I}(0)=A_{I I}=E_{z}^{\prime}(0)$. At the rod surface, the normal D field and tangential E field must be continuous, so

$$
\begin{aligned}
& \boldsymbol{E}_{z \boldsymbol{I}}(b)=\boldsymbol{E}_{z \boldsymbol{I I}}(b) \\
& \boldsymbol{E}_{\boldsymbol{r} \boldsymbol{I}}(b)=\varepsilon^{\prime} \boldsymbol{E}_{\boldsymbol{r} \boldsymbol{I}}(b)
\end{aligned} .
$$

As mentioned previously, the rod is assumed to be placed ir a snap-shot of the unperturbed fields, so the incident fields are equal to the original fields, $\gamma \approx \gamma^{\prime}$, and $\beta \approx \beta^{\prime}$ at the perturbation rod surface. With these assumptions, $E_{z 1}$ can be extended to the axis and evaluated resulting in $A$ $=A_{1}=E_{z}(0)$. The perturbed on-axis longitudinal field magnitude, $E_{z}^{\prime}(0)=E_{211}(0)=A_{11}$.

Substituting equations 11 and 12 into 13 and eliminating $B_{1}$. Lagerstrom derives the correction factor $p_{1}$ relating the perturbed and unperturbed on-axis longitudinal electric field magnitudes as 


$$
p_{1}=\frac{E_{z}^{\prime}(0)}{E_{z}(0)}=\frac{A_{I I}}{A_{I}}=\frac{I_{0}(\gamma b)}{I_{0}\left(\gamma^{\prime} b\right)} \frac{1}{1+\frac{\varepsilon^{\prime} V^{\prime}-V}{U}}
$$

where

$$
\begin{aligned}
& U=\frac{2}{(\gamma b)^{2} I_{0}(\gamma b) K_{0}(\gamma b)} \\
& V=\frac{2 I_{1}(\gamma b)}{(\gamma b) I_{0}(\gamma b)}
\end{aligned}
$$

and

$$
V^{\prime}=\frac{2 I_{1}\left(\gamma^{\prime} b\right)}{\left(\gamma^{\prime} b\right) I_{0}\left(\gamma^{\prime} b\right)}
$$

Substituting the electric field components and taking advantage of the assumption that there is no azimuthal or axial variation, equation 3 can be expressed as

$$
\Delta \beta=\frac{2 \pi \omega \varepsilon_{0}\left(\varepsilon^{\prime}-1\right) \int_{0}^{b} r\left[E_{z 0}^{\prime}(r) E^{*}{ }_{z 0}(r)+E^{\prime}{ }_{r \theta}(r) E^{*}{ }_{r 0}(r)\right] d r}{4 P} .
$$

The fields within the integral can be expressed in terms of the on-axis fields using equations 9 , 10 and 12:

$$
E_{z 0}^{\prime}(r) E_{z 0}^{*}(r)+E_{r 0}^{\prime}(r) E_{r 0}^{*}(r)=E_{z 0}^{\prime}(0) E_{z 0}^{*}(0)\left[I_{0}\left(\gamma^{\prime} r\right) I_{0}(\gamma r)+\frac{\beta \beta^{\prime}}{\gamma \gamma^{\prime}} I_{1}\left(\gamma^{\prime} r\right) I_{1}(r)\right] .
$$

Lagerstrom defines two additional correction factors: 


$$
p_{2}=\frac{2}{b^{2}} \int_{0}^{b} r I_{0}\left(\gamma^{\prime} r\right) I_{0}(\gamma r) d r=I_{0}\left(\gamma^{\prime} b\right) I_{0}(\gamma b)\left[V^{\prime}-\left(\frac{\gamma}{k}\right)^{2} \frac{V^{\prime}-V}{\varepsilon^{\prime}-1}\right]
$$

and

$$
p_{3}=\frac{2}{b^{2}} \int_{0}^{b} r I_{1}\left(\gamma^{\prime} r\right) I_{1}(\gamma r) d r=\frac{\gamma^{\prime}}{\beta^{2}} I_{0}\left(\gamma^{\prime} b\right) I_{0}(\gamma b)\left[\frac{V^{\prime}-V}{\varepsilon^{\prime}-1}\right] \text {. }
$$

To simplify the notation $p_{e}$ is defined as

$$
p_{e}=p_{1}\left(p_{2}+\frac{\beta^{2}}{\gamma \gamma^{\prime}} p_{3}\right)=\frac{1}{\varepsilon^{\prime}-1} \frac{I_{0}^{2}(\not b)}{U}+\frac{1}{\varepsilon^{\prime} V^{\prime}-V}\left[1-\left(\frac{\gamma}{k}\right)^{2} \frac{V^{\prime}-V}{\varepsilon^{\prime} V^{\prime}-V}\right]
$$

and the integral of equation 18 becomes

$$
2 \pi \int_{0}^{b} r\left[E_{z 0}^{\prime}(r) E_{z 0}^{*}(r)+E_{r 0}^{\prime}(r) E_{r 0}^{*}(r)\right] d r=\pi \zeta^{2} p_{e} E_{z 0}(0) E_{z 0}^{*}(0)
$$

Substituting equation 23 into equation 3, Lagerstrom arrives at

$$
\Delta \beta=\frac{1}{4 P} \omega\left(\varepsilon^{\prime}-1\right) \pi b^{2}\left[p_{e} \varepsilon_{o} E_{z o}(0) E_{z o}^{*}(0)\right]
$$

Consequently, the on-axis interaction impedance can be expressed in terms of the change in propagation constant as

$$
\left.K_{0}=\frac{E_{z 0}(0) E_{z 0}^{*}(0)}{2 \beta^{2} P}=\frac{1}{p_{e}} \frac{240}{\left(\varepsilon^{\prime}-1\right)} \frac{\beta}{k} \frac{\gamma}{\beta}\right)^{2} \frac{\Delta \beta}{\beta} \frac{1}{(\gamma b)^{2}} .
$$

It should be noted, as Wang and Carter [7] point out, that Lagerstrom omitted a term when evaluating $p_{e}$ (equation 3.27 of [4]). They report in [7] that this omission results in an error 
which is larger than the measurement error at higher frequencies. Equation 22 gives the correct formula.

The above analysis which applies to the nonresonant perturbation method can also be applied to the resonant perturbation method where the resonant frequencies are measured in a truncated section of circuit before and after perturbation. The change in frequency can be related to the change in axial phase shift by equation 3.54 of [4]

$$
\frac{\Delta f}{f}=-\frac{v_{g}}{v_{p}}\left(\frac{\Delta \beta}{\beta}\right)
$$

where $\beta, v_{p}$ and $f$ are the unperturbed axial phase shift, fundamental phase velocity and frequency, respectively, and $\mathrm{v}_{\mathrm{g}}$ is the group velocity of the perturbed structure.

Another common approximation made when the dielectric rod is small, is that the axial electric field is uniform in the transverse direction and the transverse electric fields can be neglected. Making these assumptions simplifies the problem to a great extent since the field tangential to the perturbing rod must be continuous through the boundary; thus, the magnitude of the perturbed field is equal to that of the unperturbed field everywhere [4]. In this case, equation 3 is easily solved and the interaction impedance is expressed as

$$
K_{0}=\frac{E_{z}(0) E_{z}^{*}(0)}{2 \beta^{2} P}=\frac{2}{\omega \beta\left(\varepsilon^{\prime}-1\right) \varepsilon_{o} \pi b^{2}} \frac{\Delta \beta}{\beta}
$$

Lagerstrom refers to equation 26 as the first order perturbation expression. He has improved upon this theory by deriving the correction factor $\mathrm{p}_{\mathrm{e}}$ to account for the transverse fields, the variation of the axial fields in the transverse direction and the difference between the perturbed 
and unperturbed fields. This first order perturbation expression is still used, however, by various researchers so it is worthwhile comparing these results with the corrected theory by Lagerstrom.

\section{SIMULATED INTERACTION IMPEDANCE}

The on-axis interaction impedance for the $\mathrm{n}^{\text {th }} \mathrm{RF}$ space harmonic as defined in equation 1 can be calculated directly using a three-dimensional computer code such as MAFIA. This study uses a method where one turn of the helical circuit is simulated. The cold-test characteristics can be obtained by applying the quasi-periodic boundary condition of MAFIA to the longitudinal ends. This feature of the code permits the user to choose a fixed phase advance per turn in the axial direction, allowing the frequency to be obtained at any axial phase shift. The interaction impedance is then calculated at this phase shift directly from equation $1 . E_{z x}(0)$ is obtained by doing a Fourier analysis on the total on-axis axial electric field, ${ }_{E_{z}} \uparrow(0)$ :

$$
E_{z n}(0)=\frac{1}{L} \int_{0}^{L} \hat{E}_{z}(0) e^{j \beta_{n} z} d z
$$

The time averaged $R F$ power flow $P$ is defined by

$$
P=\underline{w} v_{g},
$$

where $v_{g}$ is the group velocity and $w$ is the time averaged stored electromagnetic energy per unit length,

$$
w=\frac{W_{T}}{N L}
$$

with $\mathrm{W}_{\mathrm{T}}$ the total energy and $\mathrm{N}$ the number of helical turns modeled. $\mathrm{W}_{\mathrm{T}}$ is calculated directly by 
MAFIA and the group velocity is calculated by taking the slope of the dispersion curve.

\section{RESULTS}

Using the MMPM helical slow-wave circuit, simulations are compared with experimental cold-test measurements below.

\section{A. Dispersion}

The dispersion was measured experimentally by using the resonant perturbation method. An HP 8722D VNA was used to measure the resonant frequencies of the MMPM cold-test structure consisting of 159 helical turns [11]. At the helical boundaries are sections of simple cylindrical waveguide with diameter equal to that of the slow-wave circuit outer barrel and axial length about 1.4 times the length of the longest wavelength of operation. The dispersion was also calculated using MAFIA by simulating one helical turn and using quasi-periodic boundary conditions. The measured results and those obtained using MAFIA are plotted in Figure 3. The agreement is excellent with an absolute average difference across the bandwidth of less than $0.13 \%$. The CPU time for each frequency point is about 2 hours using a IBM RISC/6000 Model 595 Workstation. 


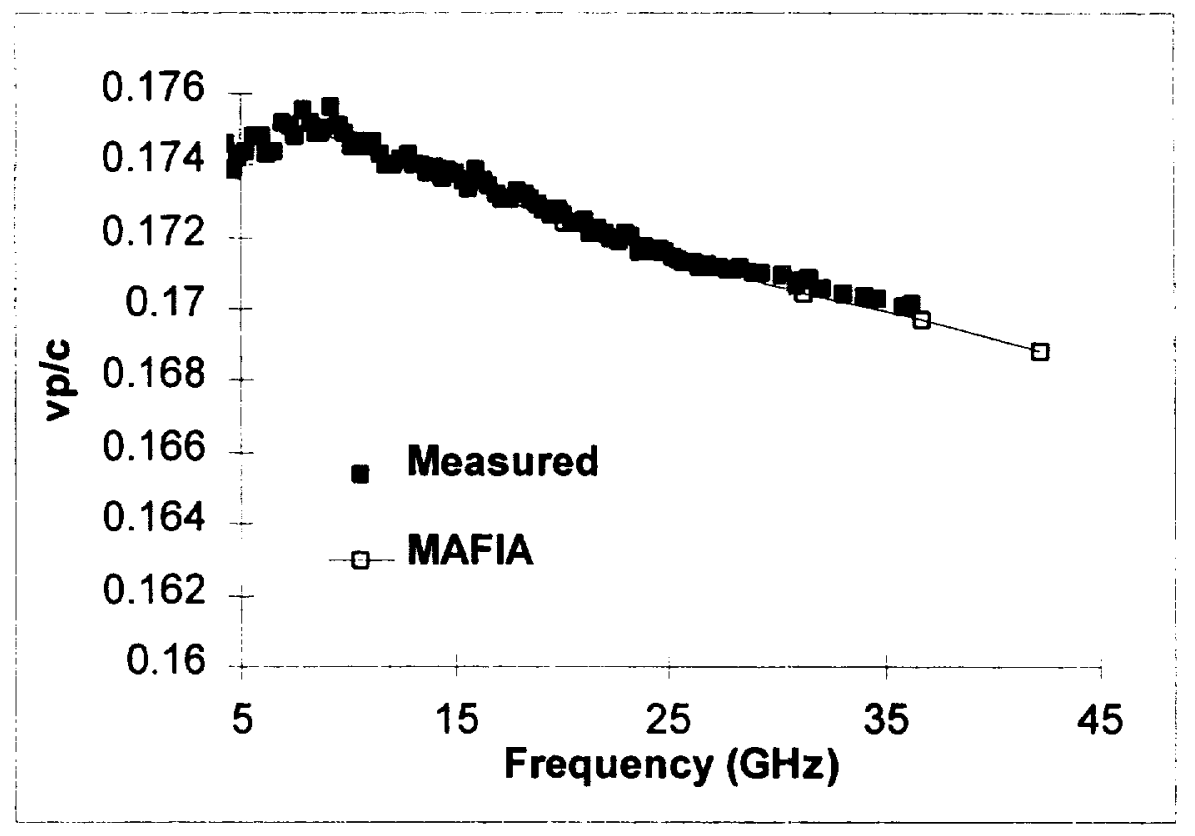

Figure 3 Measured and simulated dispersion for MMPM helical slow-wave circuit

\section{B. Interaction Impedance}

A cylindrical sapphire rod was placed on the central axis of the MMPM experimental test structure and the resonant frequencies measured again. The perturbation rod radius to average helix radius ratio is 0.5 . The precise positioning of the rod on the center of the structure was accomplished by fitting the rod into axial end holes in the test fixture with diameter slightly larger than the perturbing rod diameter. Using equations 25 and 26 , the on-axis interaction impedance was calculated and is plotted in Figure 5 along with the results from calculations using MAFIA and equation 1. The results calculated directly from equation 1 using MAFIA are consistently lower than measured results with an average difference of $26.6 \%$.

MAFIA was also used to duplicate the perturbation experiment by simulating the helical circuit with a cylindrical dielectric rod of size and material properties consistent with the experimental setup. A cross-section of the MAFIA MMPM helical circuit modeled in the 
cylindrical coordinate system with the perturbing rod is shown in Figure 4. Computationally, the applied frequency necessary to hold the phase shift constant when a perturbing rod is inserted is obtained. Using the simulated change in frequency $\Delta \mathrm{f}$, the impedance was calculated in the same manner as the experimental values using equations 25 and 26. The MAFIA perturbation interaction impedance results are also plotted in Figure 5. These results are coincident with the measured values emphasizing the accuracy of the code. Also plotted are the MAFIA perturbation results using the first order perturbation expression of equation 27 . This data shows the large discrepancy between measured results when Lagerstrom's correction factor is incorporated and when it is assumed that the axial electric field is uniform in the transverse direction and the transverse fields are neglected. With these assumptions, the interaction impedance is an average of $52 \%$ lower across the bandwidth compared to measured data using Lagerstrom's correction factor $p_{e}$.

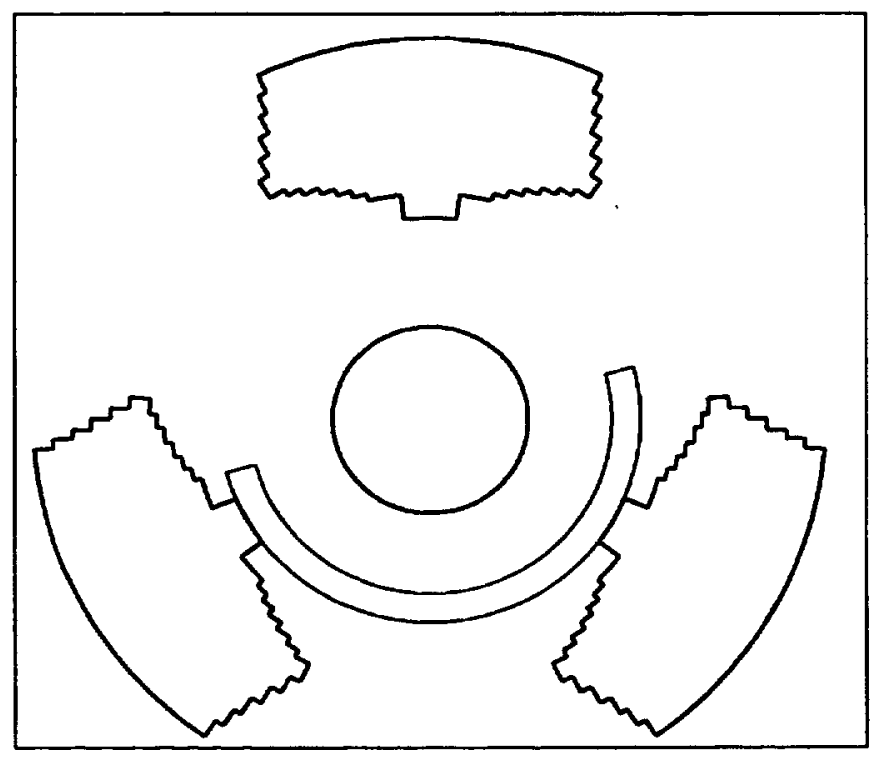

Figure 4 MAFIA cross-sectional view of MMPM helical slow-wave circuit with perturbing rod 


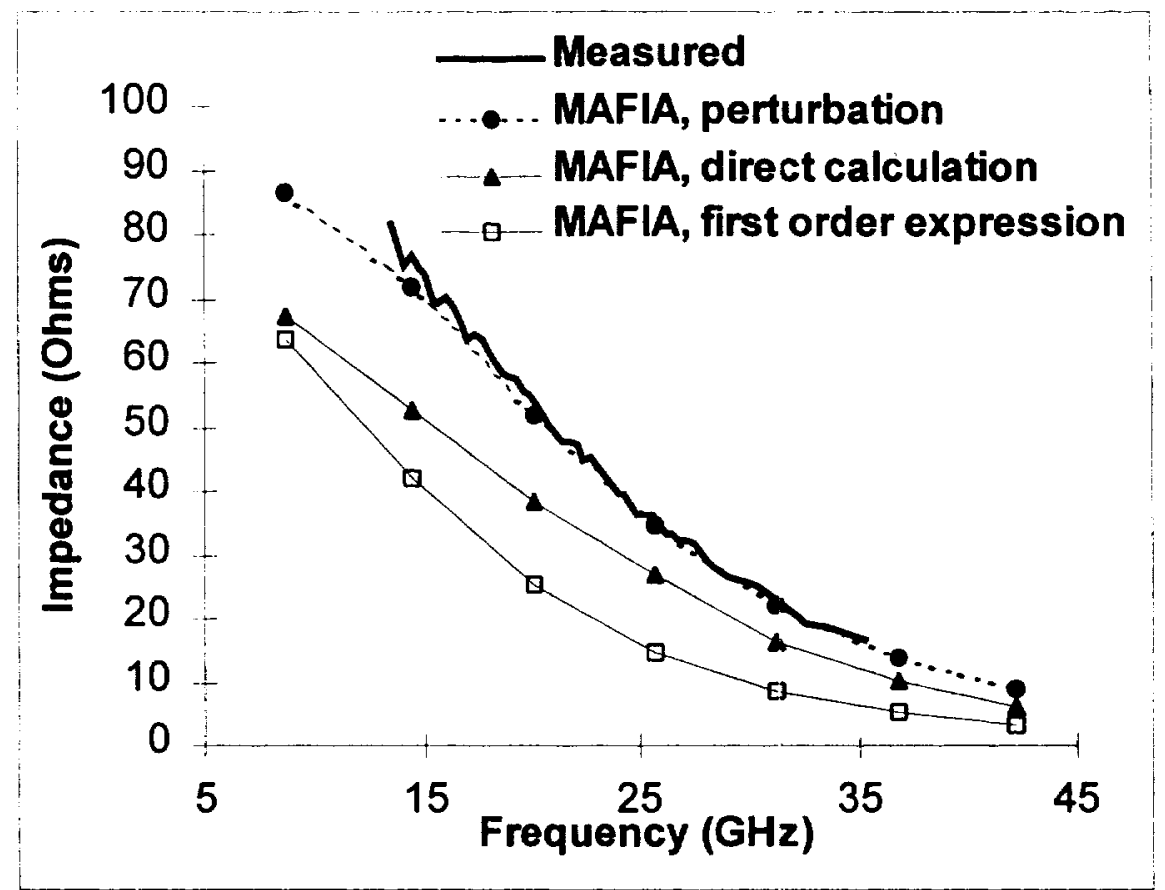

Figure 5 On-axis interaction impedance obtained by measurement and using MAFIA with the perturbation method (eqn. 25), direct calculation (eqn. 1), and the first order perturbation expression (eqn. 27)

\section{INVESTIGATION OF LAGERSTROM'S APPROXIMATIONS}

Figure 5 shows that there is a definite discrepancy between measured impedance and results which were obtained using MAFIA and directly calculating the impedance from equation 1. This plot, as well as Figure 3, verifies the accuracy of the simulated model by the outstanding agreement in the measured and simulated dispersion and impedance using the perturbation method. The purpose of this section is to investigate the major approximations made in Lagerstrom's analysis and determine their accuracy. To perform a meaningful comparison of electric fields for various simulations, it is necessary to comoare $E^{2} / w$ as each simulation is normalized to an arbitrary excitation voltage. For all of the simulations reported on in this 
report, the energy value stays constant making it valid to compare only the electric fields. It should be mentioned that using quasi-periodic boundaries with MAFIA simulates traveling waves. Therefore, the fields have amplitude and phase. In the following field comparisons, only the amplitudes are shown.

The major approximations which are made in Lagerstrom's analysis are:

A. Only the fundamental axial harmonic of $\mathbf{E}_{z}$ is present within the perturbing rod volume ( $\mathrm{n}=$ 0)

B. The fields are non-azimuthally varying within the perturbing rod volume $(v=0)$

C. The TM portion of the field dominates the TE portion over the perturbation rod volume $\left(\mathbf{H}_{\mathbf{z}}\right.$ $\left.=\mathbf{E}_{9}=0\right)$

D. The perturbed and unperturbed electric fields are equal at the perturbing rod surface $\left(\mathbf{E}_{\mathrm{II}}(\mathbf{b})=\right.$ $\left.\mathbf{E}_{\mathbf{l}}(\mathbf{b})\right)$

\section{A. Axial space harmonics}

In the analysis of [4] the axial electric fields expressed in equation 7 are assumed to contain only the fundamental axial space harmonic, $n=0$, over the perturbing rod volume. The total longitudinal electric field amplitudes at an azimuthal angle of zero versus axial distance $\mathrm{z}$ (expressed in number of turns) at radial distances of zero, half the perturbation rod radius (b/2) and the perturbation rod radius (b) are plotted in Figure 6 for different values of axial phase shift. The wide range of axial phase shifts represents a large frequency range $(8.75-42.26 \mathrm{GHz})$ so that the reader may get a qualitative idea of the space harmonic content as a function of frequency as well as radius. A Fourier analysis was done on the total longitudinal electric field along $\mathrm{z}$ at the mentioned values of radius and azimuthal angle of zero to give a quantitative 
analysis of the axial space harmonic content. The amplitudes of the $n=0, \pm 1$ and \pm 2 space harmonics are plotted in Figure 7 (additional space harmonics were not of significant amplitude). These plots show that on the axis of the circuit $(\mathrm{r}=0)$, the approximation is excellent as the non-zero space harmonic amplitudes are ins gnificant compared to the fundamental. However, at finite values of radius the amplitude of the $n=-1$ space harmonic becomes more significant, increasing with radius as one would expect. It is also seen from the plots that the amplitude of the $n=-1$ space harmonic becomes more significant with increasing frequency. The inaccuracy of this approximation in the analysis is clearly shown here as the $n=$ -1 harmonic is about $50 \%$ of the fundamental at the perturbation rod radius at about $42 \mathrm{GHz}$. These results imply that the addition of the $n=-1$ space harmonic to the measurement analysis would be sufficient to improve its accuracy.

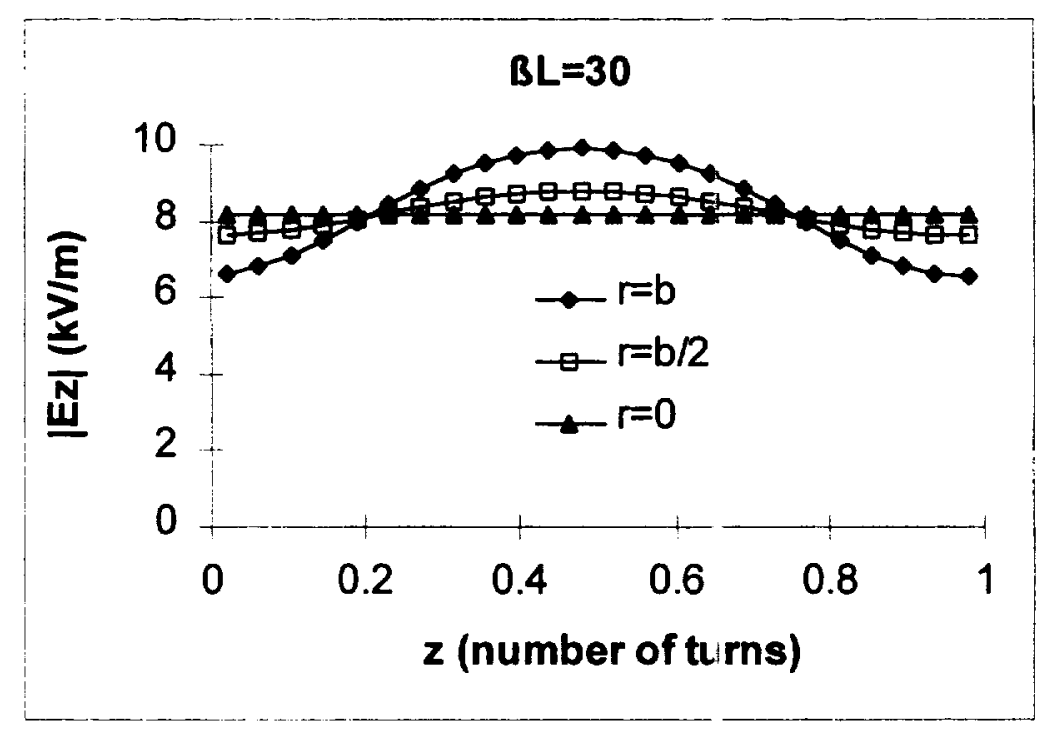

(a) 


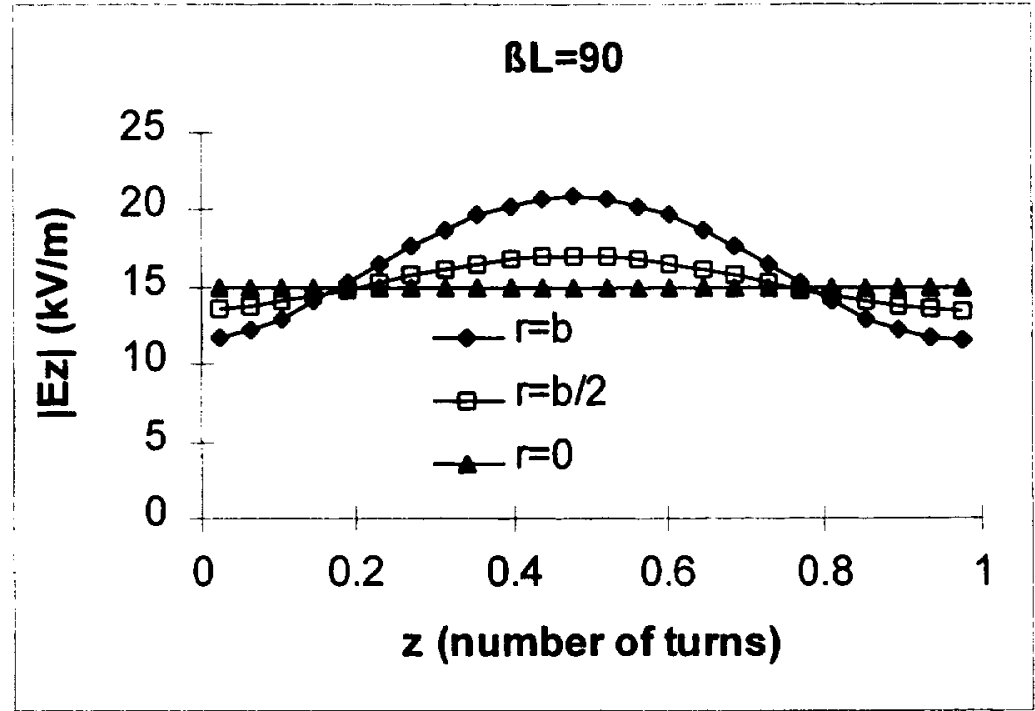

(b)

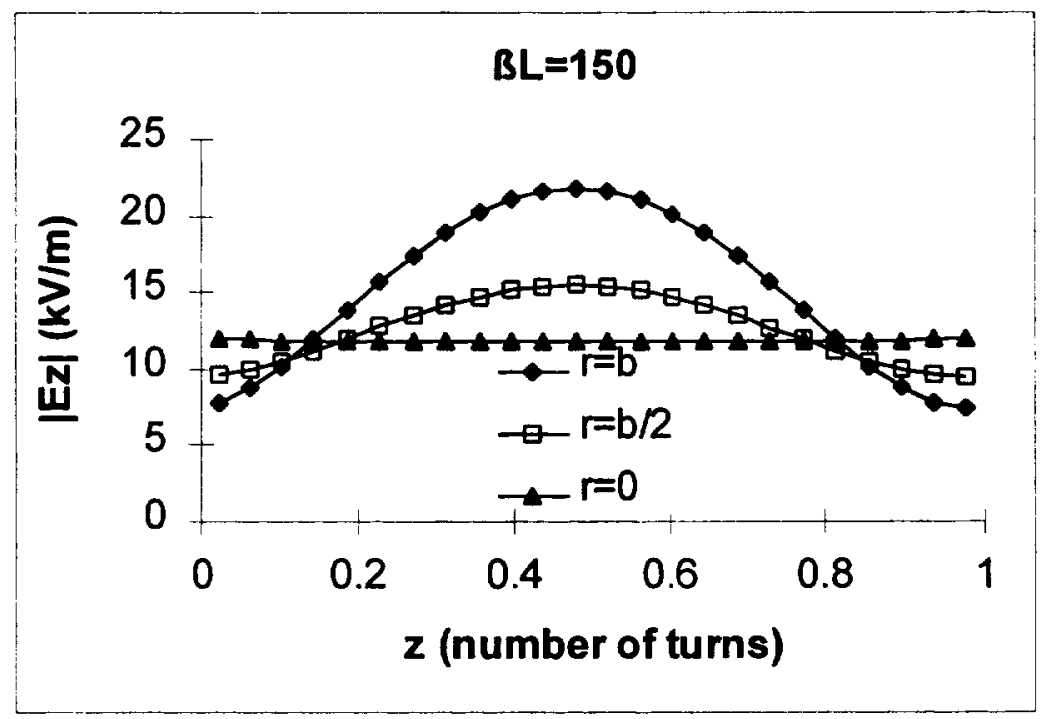

(c)

Figure 6 Total longitudinal electric field amplitude at $\theta=0$ as a function of $z$ for (a) $\beta L=30$ degrees $(8.75 \mathrm{GHz})$ (b) $\beta \mathrm{L}=90$ degrees $(25.72 \mathrm{GHz})$ and $(\mathrm{c}) \beta \mathrm{L}=150$ degrees $(42.26 \mathrm{GHz})$ 


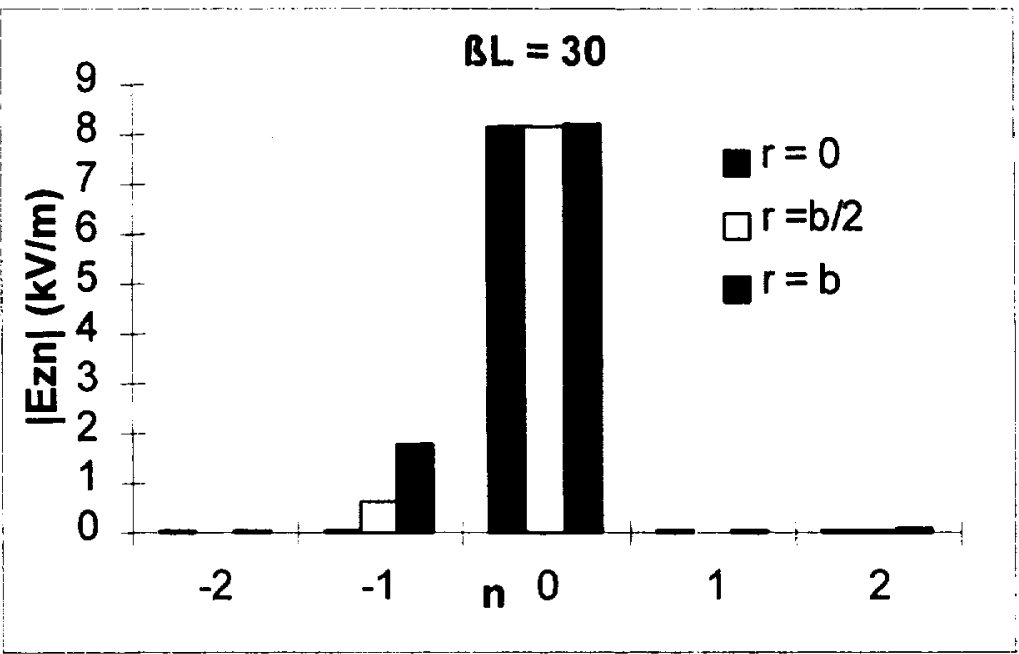

(a)

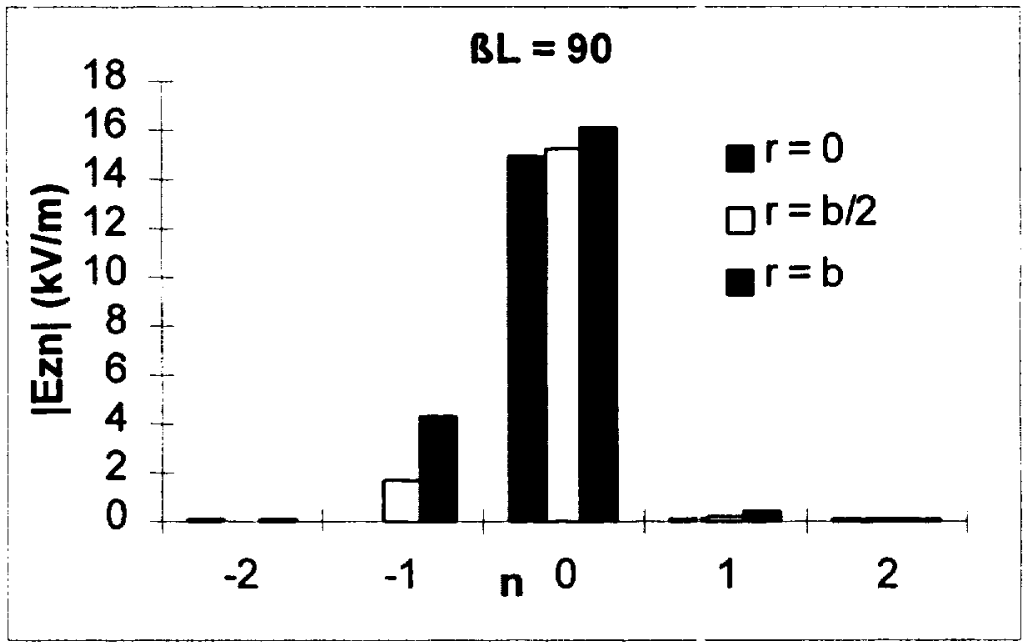

(b) 


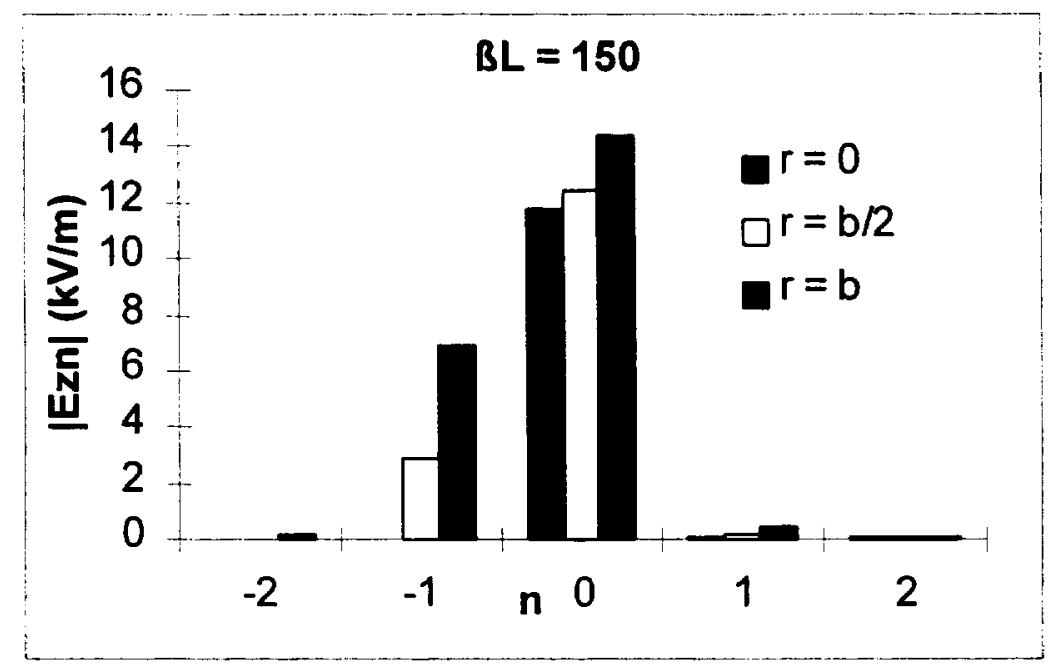

(c)

Figure 7 Magnitude of axial space harmonics of total longitudinal electric field at $\theta=0$ for (a) $\beta \mathrm{L}=30$ degrees $(8.75 \mathrm{GHz})$ (b) $\beta \mathrm{L}=90$ degrees $(25.72 \mathrm{GHz})$ and (c) $\beta \mathrm{L}=150$ degrees (42.26 GHz)

\section{B. Azimuthal space harmonics}

In the analysis of [4] the axial electric fields expressed in equation 7 are assumed to contain only the fundamental azimuthal space harmonic, $v=0$, over the perturbing rod volume. The total axial electric field amplitudes versus azimuthal distance $\theta$ (expressed in degrees) at the perturbation rod radius (b) and $z=0.5 \mathrm{~L}$ are plotted in Figure 8 for several values of axial phase shift. From these plots, it is obvious that there is indeed variation in the field with azimuthal angle. A Fourier analysis was done on the field along $\theta$ at the perturbation rod radius and $z=$ $0.5 \mathrm{~L}$ to give a quantitative analysis of the azimuthal space harmonic content. The amplitudes of the $v=0, \pm 1, \pm 2$ and \pm 3 space harmonics are plotted in Figure 9 (additional space harmonics were not of significant amplitude). These plots show that at the perturbation rod radius, the $v=-$ 1 space harmonic amplitude is significant equaling about $50 \%$ of the fundamental at $42 \mathrm{GHz}$. 
The $v=1$ space harmonic amplitude begins to emerge at higher frequencies. From inspection of Figure 7 and Figure 9, it can be seen that the $v^{\text {th }}$ space harmonic magnitudes are about equal to the $n^{\text {th }}$ space harmonic magnitudes. This verifies the common assumption that $v=n$ because of the skew symmetry of the helix [13].

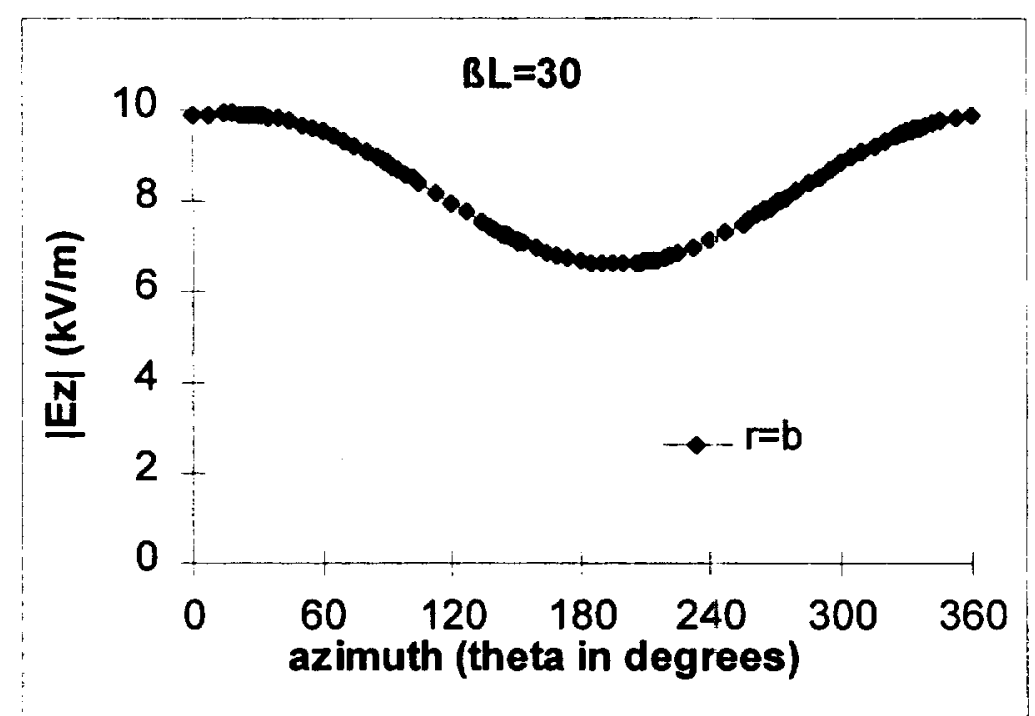

(a)

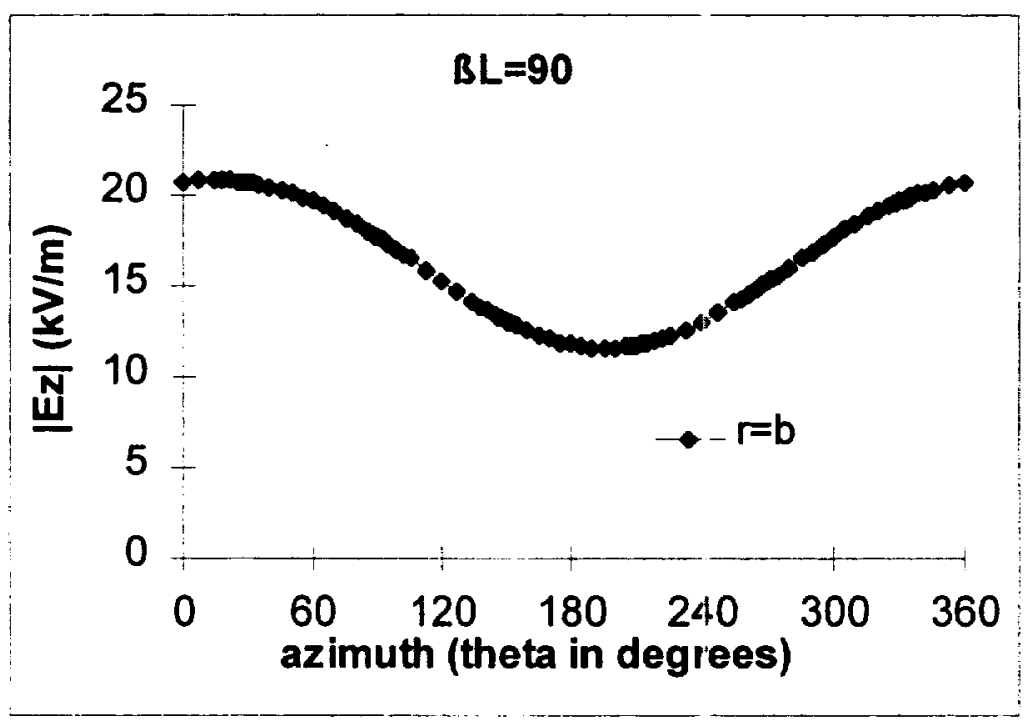

(b) 


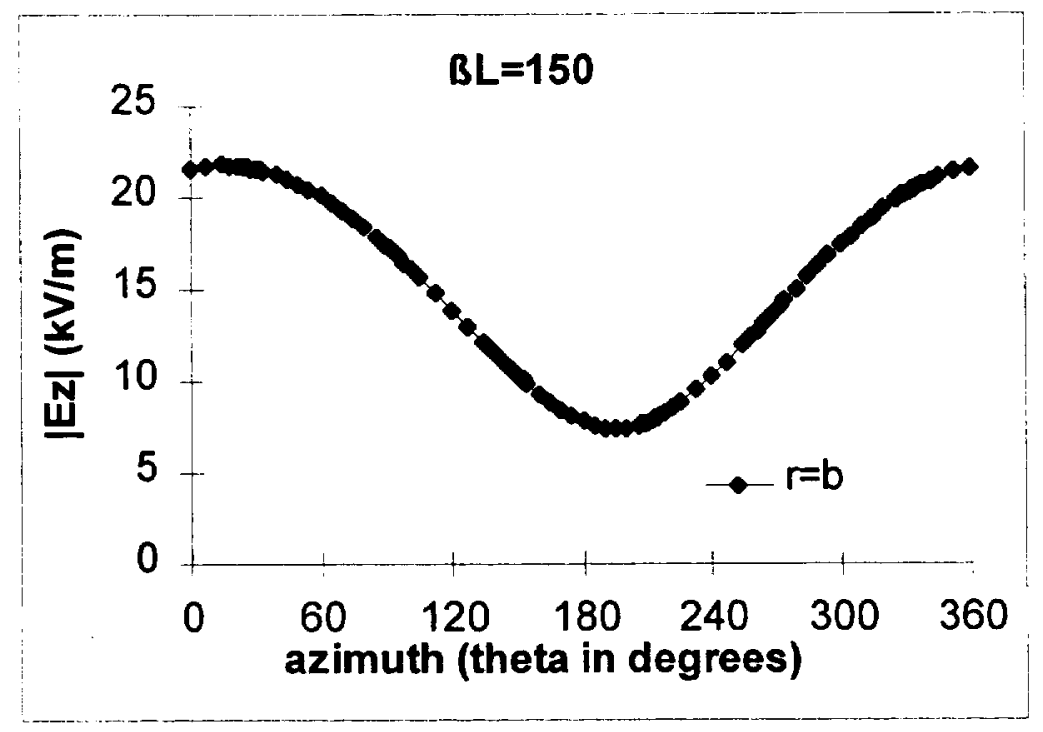

(c)

Figure 8 Total axial electric field amplitude as a function of $\theta$ at $z=0.5 \mathrm{~L}$ for (a) $\beta L=30$ degrees $(8.75 \mathrm{GHz})$ (b) $\beta \mathrm{L}=90$ degrees $(25.72 \mathrm{GHz})$ and (c) $\beta \mathrm{L}=150$ degrees $(42.26 \mathrm{GHz})$

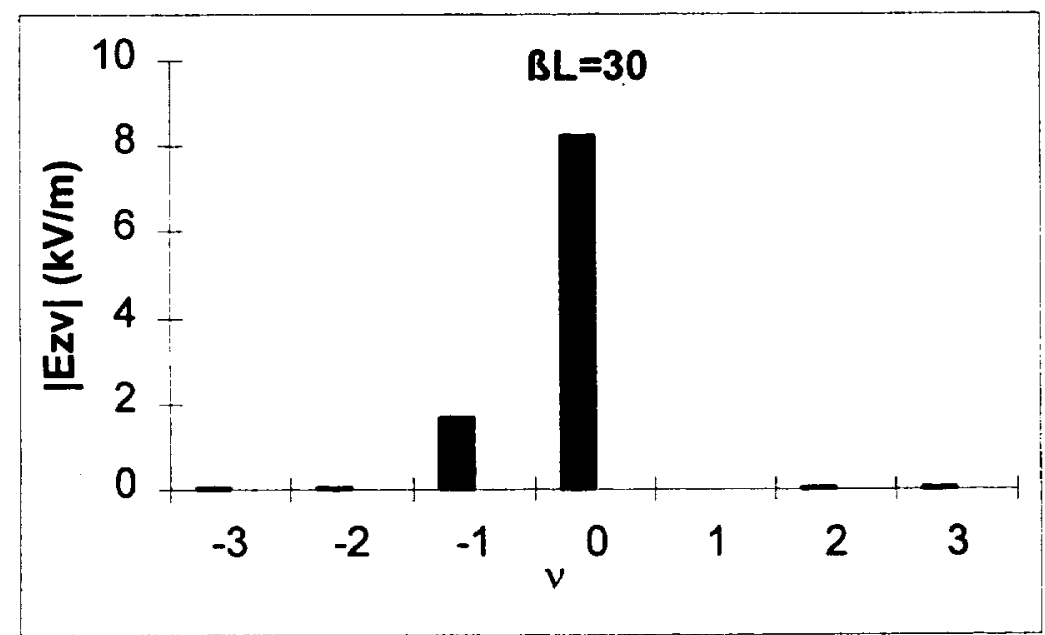

(a) 


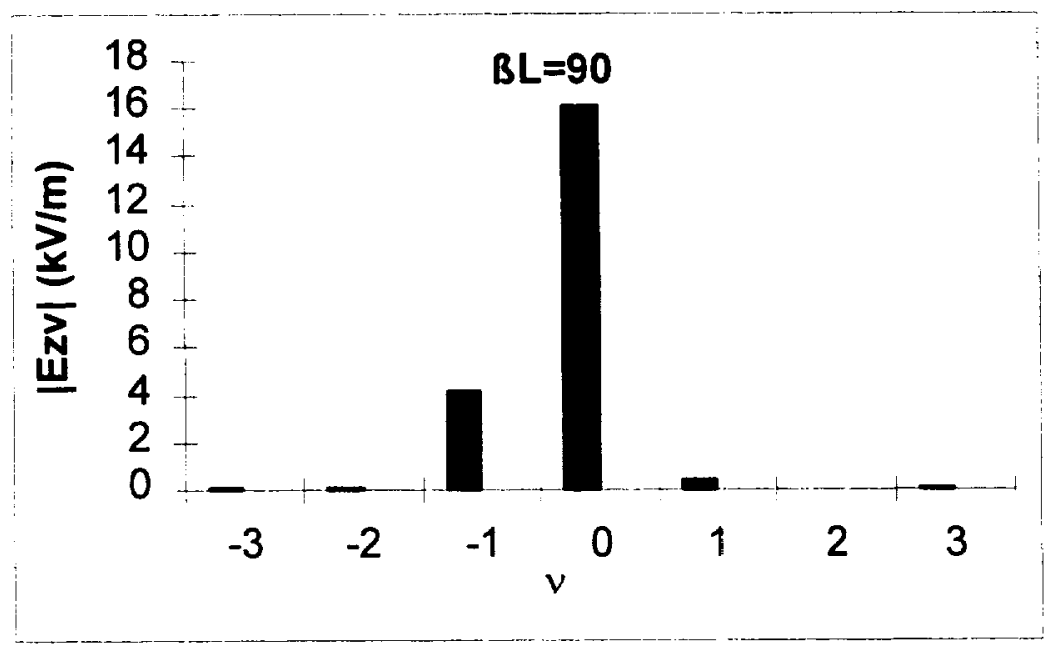

(b)

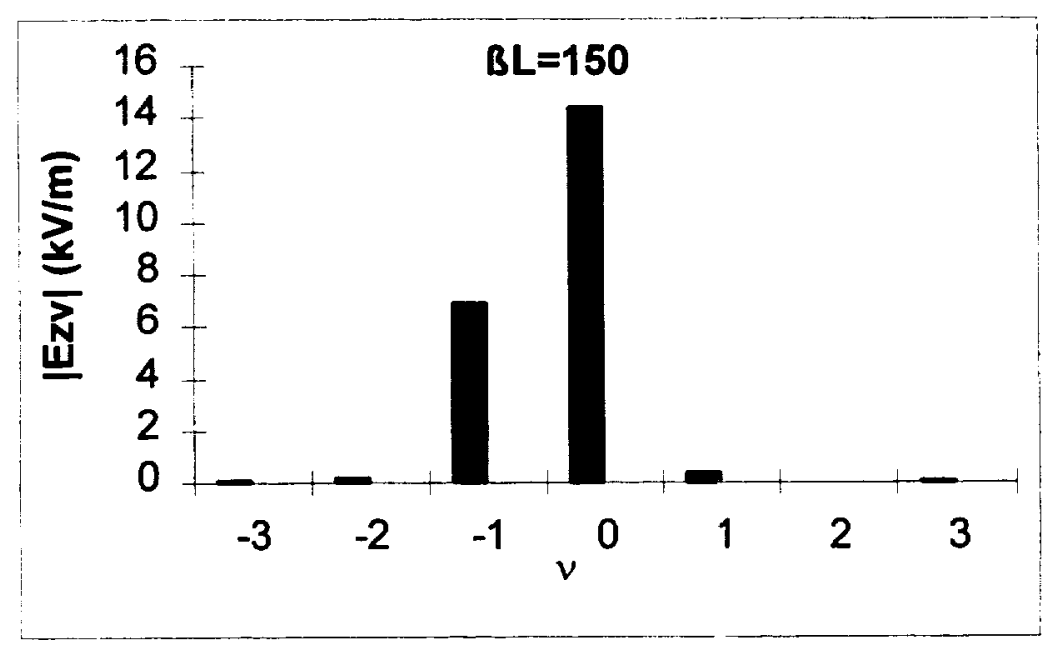

(c)

Figure 9 Magnitude of azimuthal space harmonics of total longitudinal electric field at $\mathrm{z}=$ $0.5 \mathrm{~L}$ for (a) $\beta \mathrm{L}=30$ degrees $(8.75 \mathrm{GHz})$ (b) $\beta \mathrm{L}=90$ degrees $(25.72 \mathrm{GHz})$ and (c)

$$
\beta \mathrm{L}=150 \text { degrees }(42.26 \mathrm{GHz})
$$

\section{TE Fields}

The TE portion of the fields is neglected in the integration of the fields over the perturbation rod volume. Thus, from equation 8 the azimuthal component of the electric field is 
must be zero. The azimuthal electric field amplitudes normalized to the maximum value of longitudinal electric field at the perturbation rod radius and $\theta=0$ are plotted in Figure 10 versus z. From the plot, it can be seen that $E_{\theta}$ is nontrivial amounting to about $10 \%$ of $E_{z \max }$ at $\beta \mathrm{L}=30$ degrees and about $30 \%$ at $\beta \mathrm{L}=150$ degrees.

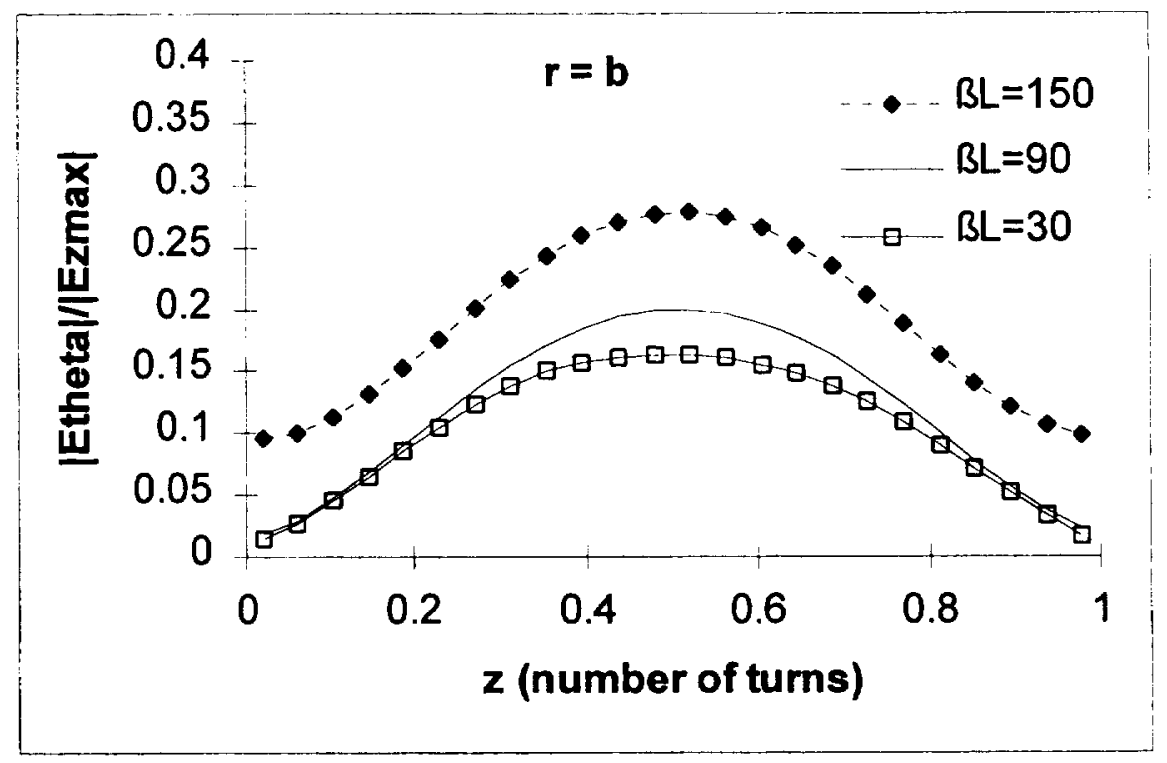

Figure 10 Azimuthal electric field amplitude normalized to the maximum value of longitudinal electric field as a function of $\mathrm{z}$ at the perturbation rod radius and $\theta$ $=\mathbf{0}$

\section{Perturbed and unperturbed fields}

In the analysis of [4] it is assumed that the perturbed and unperturbed electric fields are equal outside the perturbing rod $\left(\mathbf{E}_{\mathrm{II}}(\mathbf{b})=\mathbf{E}_{1}(\mathbf{b})\right)$. From this assumption the correction factor $\mathrm{p}_{1}$ of equation 13 is derived which relates the on-axis longitudinal electric field before and after perturbation as $E_{z}^{\prime}(0)=p_{1} E_{z}(0)$. To investigate this approximation, the magnitudes of the perturbed fields on the axis obtained directly from MAFIA simulations and as a product of the 
correction factor $p_{1}$ and the unperturbed field $E_{z}(0)$ are compared. The results are plotted in Figure 11 showing an average difference of $14 \%$ across the bandwidth.

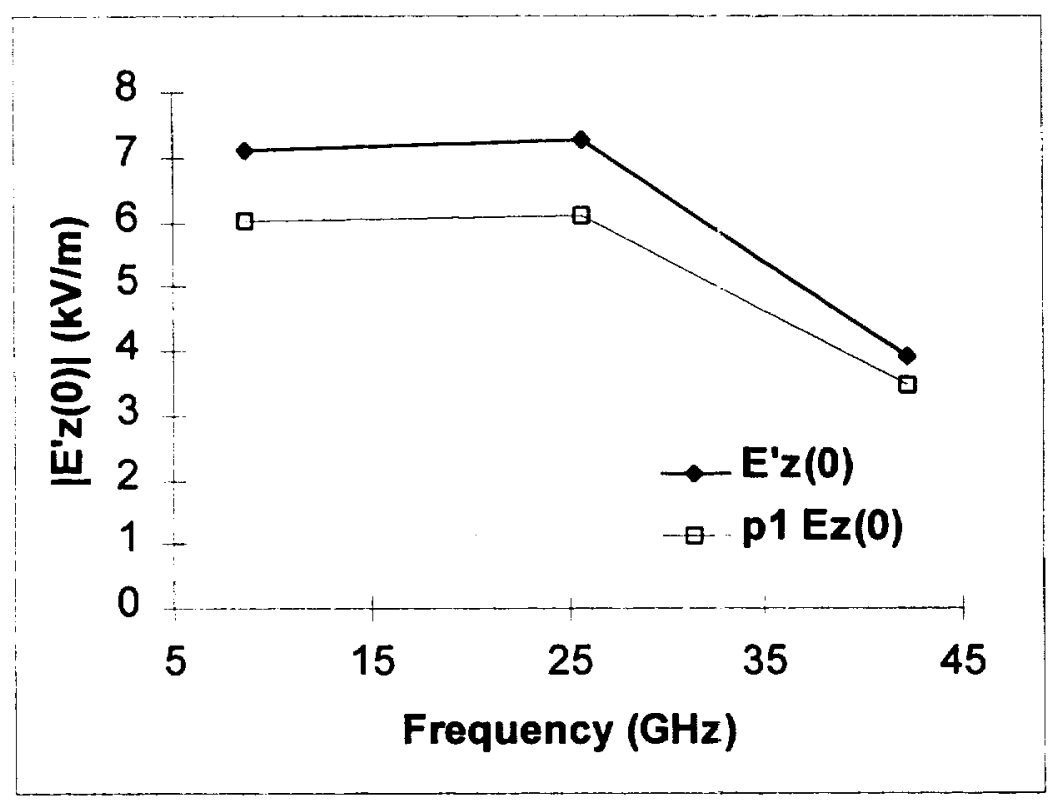

Figure 11 Perturbed on-axis longitudinal electric field amplitude obtained directly from MAFIA simulation and using Lagerstrom's correction factor $\mathbf{p}_{1}$

\section{CONCLUSIONS}

The advent of accurate three-dimensional helical circuit models has made it possible, for the first time, to investigate standard approximations involved in experimental interaction impedance calculations. A summary was given of Lagerstrom's analysis which relates change in axial phase shift or frequency between a perturbed and unperturbed helical circuit to impedance. The major approximations were emphasized and followed by an investigation of the accuracy of each assumption using MAFIA. The inaccuracy of the approximations along with the large discrepancy between measured impedance via perturbation and direct calculations using MAFIA prove that a more accurate value of interaction impedance can be obtained by using three- 
dimensional computational methods rather than performing costly and time-consuming experimental cold-test measurements. This implies a large savings in time and money associated with fabricating and testing circuits or scaled circuit models, as well as more accurate results.

These results are particularly meaningful at higher frequencies where experimental coldtesting is more time-consuming, less accurate because of necessarily tight tolerances and more difficult to perform. In addition, smaller dimensions associated with higher frequencies imply larger b/a values where the approximations in the impedance calculations become least accurate. This study also gives an analysis of field behavior in helical circuits which can be used as an aid in improving existing helical analysis codes by pointing out which approximations cause significant error.

The accuracy of the MAFIA computational helical model was validated for the MMPM helical circuit by comparing the dispersion obtained by measurement and by using MAFIA. The agreement was outstanding with less than $0.13 \%$ difference. The interaction impedance measurements using perturbation and Lagerstrom's analysis were duplicated using MAFIA and compared to measured values with negligible difference. Validation of the computational model has also been performed for several other circuit geometries and frequency ranges in [1] providing enough confidence in the simulated results to avoid experimental measurements altogether.

\section{ACKNOWLEDGMENT}

The authors would like to gratefully acknowledge Will Menninger and Bob Benton of Hughes Aircraft Company's Electron Dynamics Division for supplying circuit data and beneficial discussions regarding RF measurements. The TWT analyzed in this paper was 
developed by Hughes under contract to Raytheon as a part of the millimeter-wave power module (MMPM) program funded by the Naval Research Laboratory. 


\section{LIST OF FIGURES}

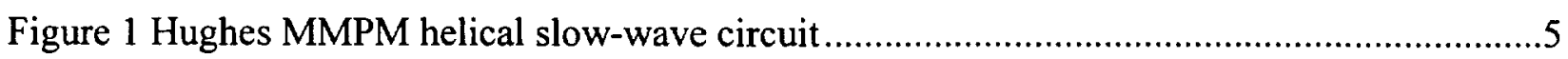

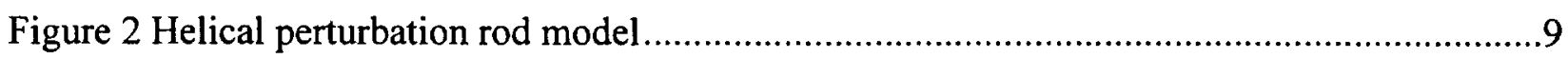

Figure 3 Measured and simulated dispersion for MMPM helical slow-wave circuit .....................16

Figure 4 MAFIA cross-sectional view of MMPM helical slow-wave circuit with perturbing rod17

Figure 5 On-axis interaction impedance obtained by measurement and using MAFIA with the perturbation method (eqn. 25), direct calculation (eqn. 1), and the first order perturbation

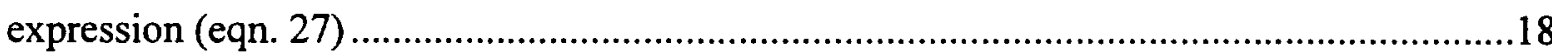

Figure 6 Total longitudinal electric field amplitude at $\theta=0$ as a function of $z$ for (a) $\beta L=30$

degrees $(8.75 \mathrm{GHz})$ (b) $\beta \mathrm{L}=90$ degrees $(25.72 \mathrm{GHz})$ and $(\mathrm{c}) \beta \mathrm{L}=150$ degrees $(42.26 \mathrm{GHz}) 21$

Figure 7 Magnitude of axial space harmonics of total longitudinal electric field at $\theta=0$ for (a) $\beta L=30$ degrees $(8.75 \mathrm{GHz})$ (b) $\beta \mathrm{L}=90$ degrees $(25.72 \mathrm{GHz})$ and $(\mathrm{c}) \beta \mathrm{L}=150$ degrees $(42.26$ $\mathrm{GHz})$ .23

Figure 8 Total axial electric field amplitude as a function of $\theta$ at $z=0.5 \mathrm{~L}$ for (a) $\beta \mathrm{L}=30$ degrees $(8.75 \mathrm{GHz})(\mathrm{b}) \beta \mathrm{L}=90$ degrees $(25.72 \mathrm{GHz})$ and $(\mathrm{c}) \beta \mathrm{L}=150$ degrees $(42.26 \mathrm{GHz}) \ldots \ldots \ldots . .25$

Figure 9 Magnitude of azimuthal space harmonics of total longitudinal electric field at $\mathrm{z}=0.5 \mathrm{~L}$ for (a) $\beta \mathrm{L}=30$ degrees $(8.75 \mathrm{GHz})$ (b) $\beta \mathrm{L}=90$ degrees $(25.72 \mathrm{GHz})$ and (c) $\beta \mathrm{L}=150$ degrees $(42.26 \mathrm{GHz})$ .26

Figure 10 Azimuthal electric field amplitude normalized to the maximum value of longitudinal electric field as a function of $\mathrm{z}$ at the perturbation rod radius and $\theta=0$

Figure 11 Perturbed on-axis longitudinal electric field amplitude obtained directly from MAFIA simulation and using Lagerstrom's correction factor $p_{1}$ 


\section{REFERENCES}

1 C. L. Kory and J. A. Dayton, Jr., Accurate cold-test model of helical TWT slow-wave circuits, IEEE Trans. on Electron Devices, in preparation.

2 K. D. Ward and J Wlodarczyk, Transmission line modeling of helix slow wave structures, 1993 IEDM Technical Digest, p. 157, IEEE 1993.

3 A. A. Mondelli, J. J. Petillo and H. P. Freund, Cold-test and interaction models for helix TWT's in MMACE, Proc. IEEE Int. Conf. on Plasma Science (ICOPS), pp. 198-199, 1997.

4 R. P. Lagerstrom, Interaction impedance measurements by perturbation of traveling waves, Stanford Electronics Laboratories Technical Report No. 7, February 11, 1957.

5. J. W. Gewartowski and H. A. Watson: Principles of Electron Tubes. D. Van Nostrand Company, Inc. p. 357, 1965.

$6 \mathrm{H}$. K. Detweiler, Characteristics of magnetically focused large-signal traveling-wave amplifiers, Rome Air Development Center Tech. Rep. RADC-TR-68-433, Griffiss Air Force Base, NY, 1968.

7 P. Wang, R. Carter and B. N. Basu, An improved technique for measuring the Pierce impedance of helix slow-wave structures, European Microwave Conference, 1994.

8 S. J. Rao, S. Ghosh, P. K. Jain, and B. N. Basu, Nonresonant perturbation measurements on dispersion and interaction impedance characteristics of helical slow-wave structures, , IEEE Trans. on Microwave Theory and Techniques, Vol. 45, No. '), pp. 1585-1593, September 1997. $9 \mathrm{~T}$. Weiland: On the numerical solution of Maxwell's equations and applications in the field of accelerator physics, Part. Accel., vol 15, pp. 245-292, 1984. 
$10 \mathrm{~T}$. Weiland: On the unique numerical solution of Maxwellian eigenvalue problems in three dimensions, Part. Accel., vol. 17, pp. 227-242, 1985.

11 Personal communication with Will Menninger of Hughes Aircraft Co., Electron Dynamics Division.

12 S. Ramo, J. R. Whinnery, and T. Van Duzen: Fields and Waves in Communications Electronics, 2nd ed. John Wiley and Sons, New York, 1984.

13 S. Sensiper, Electromagnetic Wave Propagation on Helical Conductors, Massachusetts Institute of Technology Doctoral thesis, 1951, pp.95-96. 
